\title{
Strong-field physics in the molecular frame
}

\author{
Andrea Trabattoni ${ }^{1,2}$, Sebastian Trippel ${ }^{1,2}$, Umberto De Giovannini ${ }^{3,4}$, Jean-François \\ Olivieri $^{1}$, Joss Wiese ${ }^{1,5}$, Terry Mullins ${ }^{1}$, Jolijn Onvlee ${ }^{1}$, Sang-Kil Son ${ }^{1,2}$, Biagio Frusteri ${ }^{4}$, \\ Angel Rubio ${ }^{3,6,7}$, and Jochen Küpper*1,2,5,7 \\ ${ }^{1}$ Center for Free-Electron Laser Science, Deutsches Elektronen-Synchrotron DESY, Notkestraße 85, \\ 22607 Hamburg, Germany \\ ${ }^{2}$ The Hamburg Center for Ultrafast Imaging, Universität Hamburg, Luruper Chaussee 149, 22761 \\ Hamburg, Germany \\ ${ }^{3}$ Max Planck Institute for the Structure and Dynamics of Matter and Center for Free-Electron Laser \\ Science, 22761Hamburg, Germany \\ ${ }^{4}$ Dipartimento di Fisica e Chimica, Universitá degli Studi di Palermo, Via Archirafi 36, I-90123, \\ Palermo, Italy \\ ${ }^{5}$ Department of Chemistry, Universität Hamburg, Martin-Luther-King-Platz 6, 20146 Hamburg, \\ Germany \\ ${ }^{6}$ Center for Computational Quantum Physics (CCQ), The Flatiron Institute, 162 Fifth Avenue, New \\ York NY 10010 \\ ${ }^{7}$ Department of Physics, Universität Hamburg, Luruper Chaussee 149, 22761 Hamburg, Germany
}

\begin{abstract}
Laser-aligned carbonyl-sulfide molecules were strong-field ionized using mid-infrared light. Investigating the strong-field effects in the molecular frame allowed to add novel facets to the understanding of the intrinsic nature of strong-field physics.
\end{abstract}

\section{Introduction}

The interaction of strong laser fields with matter intrinsically enables the imaging of transient dynamics with extremely high spatiotemporal resolution [1]. This paradigm of photophysics has grown into new emerging research areas, ranging from attosecond science to laser-induced electron diffraction, providing new insight into atoms, molecules and, more recently, condensed matter. Also, the earliest moments of strong-field interactions have attracted attention for capturing the intrinsic nature of strong-field physics. While pioneering attosecond-science experiments and molecular-frame measurements $[2,3]$ revealed non-trivial spatiotemporal features in electron tunneling, these initial conditions are generally considered a weak perturbation.

Here, we investigated strong-field ionization of strongly aligned molecules. We demonstrated that the electron dynamics is properly understood only in the molecular frame. Our findings have strong impact on the interpretation of photoelectron holography $[3,4]$ in molecules and allows one to reach a deeper understanding of the strong-field interaction with molecular potentials.

\section{Results and discussion}

Fig. 1 shows the experiment. An ultracold ensemble of carbonyl-sulfide molecules (OCS) was adiabatically aligned in the laboratory frame, with $\left\langle\cos ^{2} \theta_{2 \mathrm{D}}\right\rangle=0.9$, by using a linearly polarized, $500 \mathrm{ps}$ laser pulse, centered at $800 \mathrm{~nm}$.

* Corresponding author: jochen.kuepper@desy.de 
a
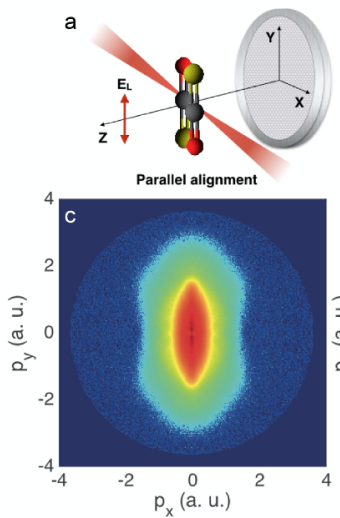

Fig.1: $\mathbf{a}, \mathbf{b}: \quad$ Sketch

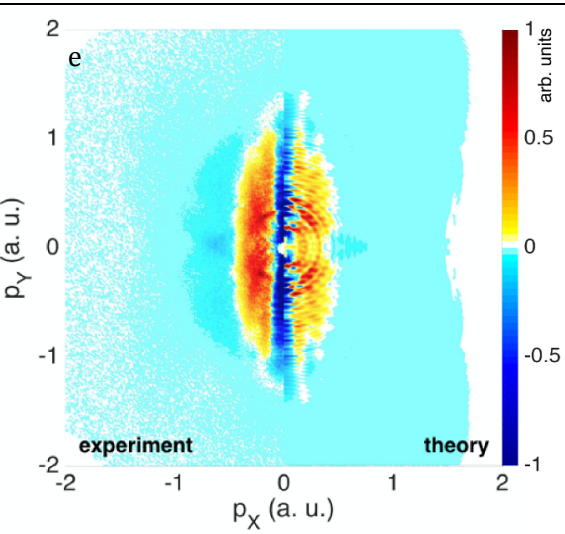

arrangement with alignment of OCS molecules in the laboratory frame, parallel and perpendicular to the $X Y$ plane. The ionizing laser was linearly polarized along the $Y$ axis and the detection was in the $X Y$ plane. c,d: Molecular-frame angle-resolved photoelectron spectra projected onto a $2 \mathrm{D}$ detector in a velocity map imaging spectrometer on a logarithmic scale. e: Measured (left) and computed (right) differential momentum distribution (parallel - perpendicular).

The molecules were aligned in two different configurations, shown in Fig.1 a,b, with the molecular axis along the $\mathrm{Y}$ and $\mathrm{Z}$ axes, named parallel and perpendicular alignment, respectively. A second laser pulse, centered at $1300 \mathrm{~nm}$, with a duration of $65 \mathrm{fs}$, and a peak intensity of $8 \cdot 10^{13} \mathrm{~W} / \mathrm{cm}^{2}$, was used to singly ionize the OCS molecules. The ionizing laser pulse, $\mathrm{E}_{\mathrm{L}}$ in Fig.1 a,b, was linearly polarized along the $\mathrm{Y}$ axis. The produced molecularframe angle-resolved photoelectron spectra (MF-ARPES) were recorded in a velocity map imaging (VMI) spectrometer with its detector parallel to the XY plane (Fig.1 c,d).

In order to have a close comparison between the two different alignment cases, we performed a differential analysis by subtracting the two experimental spectra from each other. In Fig. 1 e (left half) the relative normalized difference, parallel minus perpendicular, is reported. Thus, positive signal in Fig. 1 e corresponds to a larger contribution from parallel alignment, while negative signal refers to a larger contribution from perpendicular alignment. A strong depletion along the vertical axis and two transversely offset broad lines of positive yield appear as main features. The depletion along the centerline is due to the node along the molecular axis of the degenerate $\Pi$ highest occupied molecular orbital (HOMO) of OCS; it represents a forbidden direction of electron ejection [5]. The offset broad lines, instead, correspond to a larger transverse initial momentum, along the $\mathrm{X}$ axis, of photoelectrons in the case of parallel alignment. On top of these observations, a rich radial interference pattern was observed for both negative and positive signals. This pattern, showing the period of the ionizing photon energy, could be assigned to well-known abovethreshold ionization (ATI) features [6].

The experimental graph in Fig. 1 e also showed a weak periodic pattern of vertical fringes evolving along the $X\left(0<p_{X}<1\right.$ a.u. $)$ axis and centered at $p_{Y}=0$. These fringes appeared only in the case of perpendicular alignment (negative yield in Fig.1 e).

To unravel the experimental observations, state-of-the-art calculations were performed using time-dependent density-functional theory (TDDFT) [7]. The right half of Fig.1 e showed the normalized difference for the computed spectra. The calculation reproduced the depletion along the centerline, as well as the offset broad lines. The numerical result also showed the vertical fringes centered at $\mathrm{p}_{\mathrm{Y}}=0$, in excellent agreement with the experimental 
data. A Fourier analysis of this pattern, both for the experiment and the calculation, demonstrated that the periodicity of the fringes deviates from the photon energy spacing. Thus, it can't be assigned to ATI features. Furthermore, Fig.1 d showed that these fringes appeared on top of a fork-like structure, previously assigned to rescattered-electron trajectories for atomic systems [4]. Further simulations are required to isolate the specific trajectories contributing to the observed pattern.

In this work we demonstrated that the molecular frame is strongly trajectory selective and represents an excellent tool to investigate the electron dynamics following the strongfield interaction in molecules with high precision. This result has a strong impact in the interpretation of all experiments based on molecular photoelectron imaging and, in principle, allows one to achieve a deeper understanding of the interaction between strong laser fields and molecular potentials.

[1] P. B. Corkum and F. Krausz, “Attosecond science”, Nat. Phys. 3, 381387 (2007).

[2] P. Eckle, A. N. Pfeiffer, C. Cirelli, A. Staudte, R. Dörner, H. G. Müller, M. Buttiker, and U. Keller, "Attosecond ionization and tunneling delay time measurements in helium", Science 322, 15251529 (2008).

[3] M Meckel, A Staudte, S Patchkovskii, D M Villeneuve, P B Corkum, R Dörner, and M Spanner, "Signatures of the continuum electron phase in molecular strong-field photoelectron holography", Nat. Phys. 10, 594600 (2014).

[4] Y. Huismans, A. Rouzèe, A. Gijsbertsen, J. H. Jungmann, A. S. Smolkowska, P. S. W. M. Logman, F. Lèpine, C. Cauchy, S. Zamith, T. Marchenko, J. M. Bakker, G. Berden, B. Redlich, A. F. G. van der Meer, H. G. Muller, W. Vermin, K. J. Schafer, M. Spanner, M. Yu. Ivanov, O. Smirnova, D. Bauer, S. V. Popruzhenko, M. J. J. Vrakking, "Time-Resolved Holography with Photoelectrons", Science 331, 61-64 (2011).

[5] L. Holmegaard, J. L. Hansen, L. Kalhøj, S. L. Kragh, H. Stapelfeldt, F. Filsinger, J. Küpper, G. Meijer, D. Dimitrovski, M. Abu-samha, C. P. J. Martiny, and L. B. Madsen, "Photoelectron angular distributions from strong-field ionization of oriented molecules", Nat. Phys. 6, 428 (2010).

[6] W. Becker, F. Grasbon, R. Kopold, D.B. Miloševic, G.G. Paulus, and H. Walther, "Abovethreshold ionization: from classical features to quantum effects", Adv. At. Mol. Opt. Phys. 48, 35 (2002).

[7] M. A. L. Marques, N. T. Maitra, F. Nogueira, E. K. U. Gross, and A. Rubio, "Fundamentals of Time-Dependent Density Functional Theory”, Lecture Notes in Physics (Springer Verlag, 2011). 